INRA Prod. Anim., 2003, 16 (4), 289-293

\section{BARJOLLE ${ }^{1}$, B. SYLVANDER ${ }^{2}$}

${ }^{1}$ ETHZ - Institut d'Economie Rurale, Zürich \& SRVA - Service Romand de Vulgarisation Agricole, Jordils 1, CP 128, CH-1000 Lausanne 6

\section{d.barjolle@srva.ch}

2 INRA, UR Économie des qualifications agro-alimentaires, 8 avenue René Laennec, 72000 Le Mans

sylvander@lemans.inra.fr

\title{
Facteurs de succès des produits d'origine certifiée dans les filières agro-alimentaires en Europe : marché, ressources et institutions
}

Les produits d'origine certifiée établissent une différence par rapport aux produits standards présents sur le même marché. La notion de différenciation proposée par l'économie industrielle rejoint un premier sens de la notion de spécificité : le produit est dit différencié s'il a des caractéristiques particulières (mesurables, au sens de substantielles ou d'intrinsèques) et s'il est perçu comme tel par les usagers. Le concept économique de marché pertinent délimite les produits substituables aux yeux des consommateurs.

Deux approches de la typicité ont été proposées : une «typicité 1», horizontale, qui signifie que le bien est à la fois spécifique (différent) et unique, et donc qu'il spécifie une région donnée (on dit «typique de ...»), et une «typicité 2», verticale, qui complète la précédente en mettant l'accent sur les déterminants de la typicité 1, c'est-à-dire sur la combinaison de facteurs naturels et humains (Salette 1997). Le fait que ces derniers soient liés au savoir-faire de l'homme, relié de manière peu séparable aux facteurs naturels (Bertrand 1975), peut conduire à avancer qu'ils ne sont pas facilement reproductibles : si le savoir peut être transmissible (dans le temps) à certaines conditions, il n'est pas aisément transférable (dans l'espace) (Casabianca et De Sainte Marie 1997). En ce sens, la notion revêt un contenu culturel certain. On pourra alors définir le terroir comme une zone homogène et délimitée, où les conditions de la typicité 2 sont réunies.

Pour qu'un système d'acteurs puisse construire une filière performante dans un environnement fortement concurrentiel et mondialisé, un certain nombre de conditions doivent être réunies. Certaines sont liées au processus de mobilisation des ressources de ce système (qui peut être désigné sous le terme d'accord local), d'autres aux politiques publiques et à leur application (accord global).

L'importance de la nécessité d'une généralité dans la définition d'un bien, avancée par Thévenot (1995) et soutenue par Allaire (1995), rend indissociables ces deux moments. Certes, la généralité peut être atteinte par une politique de marque forte et de long terme, appliquée par une grande entreprise. Cependant, les produits d'origine certifiée sont souvent élaborés dans des régions défavorisées, par des réseaux de petites entreprises qui ont peu de moyens de faire valider globalement leurs efforts sans le secours d'une politique publique qui permette au compromis entre l'industrie et l'artisanat de se faire (Sylvander et Marty 1999) et d'être protégé internationalement.

Notre thèse est donc que le processus collectif de création de valeur est bien un facteur essentiel, qui doit cependant être conforté par une politique publique d'accompagnement adaptée. Nos conclusions sont issues d'une recherche effectuée dans le cadre d'un projet de recherche cofinancé par la Commission Européenne et l'Office Fédéral suisse de l'Education et de la Science (programme FAIR), intitulé «PDO - PGI products: market, supply chains and institutions» ${ }^{(1)}$. Le but de ce projet était l'analyse des conditions économiques et institutionnelles pour le développe-

(1) Les partenaires suivants étaient associés : INRA - Unité de Recherche sur l'économie des qualifications agro-alimentaires, France ; CRITT - Crisalide, France ; Centro Ricerche Produzioni Animali S.p.a., Reggio Emilia, Italy ; National Agriculture Research Foundation, Department of Marketing and Marketing Research, Greece ; Wageningen University, Holland ; Food Industry Management, Wye College Great Britain ; Institut d'Economie Rurale de l'EPFZ, Suisse. 
ment des AOC (appellations d'origine contrôlée) et des IGP (indications géographiques protégées) en Europe (règlement UE 2081/92). Des recherches ont été réalisées dans 21 filières où les produits sont enregistrés au niveau européen en AOC ou en IGP.

\section{1 / Méthodologie}

Afin d'identifier les principaux facteurs de succès des produits AOC et IGP, nous avons opté pour une approche croisée. Dans un premier temps, nous avons calculé le score de chaque critère pour chacun des produits (succès calculé). Dans un second temps, nous avons estimé le succès d'après quatre déterminants de la performance du produit sur son marché (succès observé). Nous partons du principe que si le succès calculé et le succès observé sont corrélés, les facteurs de succès ont été correctement identifiés.

\section{1 / Facteurs relatifs à l'offre et à la demande}

\section{a / Spécificité du produit}

La spécificité du produit implique un certain nombre de conditions (Sylvander et Lassaut 1994). Le produit doit avoir des caractéristiques mesurables qui sont véritablement différentes de celles des produits de substitution, classées en deux catégories distinctes :

- les caractéristiques discernables et mesurables que le consommateur peut identifier lorsqu'il achète et consomme le produit fini ;

- les caractéristiques non discernables : caractéristiques intrinsèques et caractéristiques liées aux modes de production.

Le produit doit être considéré comme différent par le consommateur. La technologie de fabrication doit être différente de celle des produits de substitution. Dans le cas des produits AOC et IGP, la technologie doit mettre en évidence le lien entre les caractéristiques finales du produit et le terroir et renforcer ce que nous appelons la typicité. La dénomination utilisée pour le produit doit se distancier de manière significative de celle utilisée pour le produit standard.

\section{b / Pertinence : le produit spécifique correspond à une demande sur un marché défini}

Le succès d'un produit spécifique dépend souvent de l'identification correcte du marché sur lequel il sera vendu, i.e. le marché pertinent. Par exemple, dans le cas du Parmigiano Reggiano, le marché pertinent est plus celui des ingrédients culinaires que celui du fromage. De la même manière, le marché pertinent de l'Huile d'olive de Nyons n'est pas le marché des huiles d'olive en général, mais plutôt le marché des produits fermiers achetés par les vacanciers.

La pertinence du marché peut être mesurée à partir de trois facteurs :

- l'attraction créée par le produit auprès des consommateurs ; elle est directement liée aux caractéristiques spécifiques du produit. Elle dépend des attentes des consommateurs et de la manière dont le produit est capable de satisfaire ces attentes ;

- une propension à payer du consommateur significative. Elle peut être mesurée par des études consommateurs (Van Ittersum 1999);

- un système de distribution adapté aux consommateurs visés. La sélection d'un mode de distribution adapté aux clients est un des principaux facteurs d'évaluation de la pertinence du marché.

\section{2 / Facteurs relatifs à l'organi- sation interne}

Nous avons ensuite formulé l'hypothèse selon laquelle le succès des produits et des filières AOC est lié de façon déterminante à la capacité des entreprises à gérer le produit à un niveau collectif. Pour vérifier cette hypothèse, nous avons considéré : le groupe d'acteurs et la motivation des entreprises ; la coordination et la coopération entre les entreprises au niveau de la gestion du produit ; la coordination et la coopération entre les entreprises au niveau de la gestion du marché ; la légitimité et l'efficacité du soutien institutionnel, local, régional et national.

\section{a/Motivation des acteurs : nécessité de se différencier et de se protéger dans une région précise}

L'équilibre entre différents groupes d'intérêts au sein de la filière est un élément important pour la définition des objectifs communs. Dans les filières étudiées, nous avons mis en évidence trois types d'entreprises :

- l'initiateur qui le premier a senti le besoin de protéger la désignation ou de mieux différencier le produit à un niveau collectif ;

- l'interprofession, c'est-à-dire le groupement demandeur (au sens du Règlement 2081/92) qui cherche une protection légale sous AOC ou sous IGP ;

- le «channel captain», c'est-à-dire le leader économique de la filière (celui qui a le pouvoir de négociation commercial le plus important).

\section{b / Coordination et coopération entre les entreprises pour la gestion du produit}

Pour mesurer l'efficacité de la coordination et de la coopération dans la gestion du produit, nous avons considéré deux facteurs principaux : la capacité de mettre en évidence le potentiel de différenciation du produit et la facilité avec laquelle chaque opérateur peut intégrer dans son entreprise les choix faits collectivement.

\section{c / Coordination et coopération entre les entreprises pour la commercialisation}

Plusieurs fonctions, généralement remplies par des entreprises individuelles, peuvent être exécutées en commun si les entreprises y trouvent un intérêt (Richardson 1972). Les principales fonctions de coordination sont : 
- le management de la qualité des matières premières : caractéristiques des matières premières en fonction du produit final désiré ;

- la définition du produit (cahier des charges) de façon cohérente par rapport aux objectifs de marché et de différenciation du produit ;

- la mise en ouvre du cahier des charges et l'évaluation du produit final en fonction du marché ;

- le paiement des matières premières en fonction de la qualité finale du produit ;

- la promotion et la gestion du signe collectif ou de la marque ;

- la gestion des volumes et de la croissance : système de contrôle de l'offre ;

- la recherche, le développement et la formation.

Ces fonctions doivent être clairement évaluées et finalisées ; la coopération ne bénéficie pas forcément à tous les acteurs de la filière.

Au plan collectif, pour l'ensemble des entreprises, la question du degré de cohérence est primordiale : une politique de promotion, par exemple, ne fonctionnera pas et sera même contre-productive si le produit est mal différencié, peu spécifique ou encore de qualité irrégulière et insuffisante. L'évaluation de la qualité n'est efficace que si le paiement de la matière première dépend directement de l'évaluation du produit final selon les critères de qualité définis dans le cahier des charges (le but étant d'obtenir un produit final de la meilleure qualité possible).

Un point important est la nature de la relation entre la gestion collective et la liberté de chaque entreprise. Le marché doit être suffisamment segmenté et compétitif pour permettre aux entreprises et au système d'évoluer. Chaque entreprise est supposée avoir un espace de liberté pour se différencier et adapter la qualité de son produit à sa propre stratégie (Marty 1998). Ces marges de manœuvre permettent aux entreprises de se faire concurrence sur des marchés segmentés (Lassaut 1997, Lassaut et al 1997).

\section{d/Légitimité et efficacité du soutien institutionnel}

Dans les pays où une législation comparable au Règlement 2081/92 existait déjà avant l'entrée en vigueur du règlement européen (France, Italie, Espagne), les autorités régionales et nationales ont souvent apporté leur soutien aux initiatives liées à la réservation des dénominations. Ce soutien peut exister sous différentes formes : financement des activités pendant la phase d'enregistrement, présence d'un comité de pilotage, soutien aux entreprises individuelles ou aux groupements demandeurs (interprofessions).

\section{3 / L'évaluation de la performance}

La profitabilité n'a pas pu être évaluée de manière directe à une large échelle. Nous nous sommes limités à l'évaluation du succès que nous comprenons comme la conjonction d'au moins trois des critères suivants : chiffre d'affaires significatif et importance économique ; taux de croissance élevé (plus important que celui du marché de référence) ; notoriété du nom du produit spécifique ou de la marque collective ; différence de prix positive par rapport au produit substitut le plus proche.

En ce qui concerne les performances sociales, nous examinons le potentiel du produit AOC ou IGP pour stimuler l'emploi dans les zones rurales.

\section{2 / Résultats}

\section{1 / Succès économique}

Pour le succès calculé, les quatre facteurs présentés plus haut ont été retenus :

- l'urgence, qui reflète la motivation des entreprises pour mettre en place elles-mêmes un système de différenciation et de protection de la dénomination ;

- la spécificité, qui fait référence à la différence objective du produit par rapport aux substituts ;

- la pertinence, qui reflète l'attractivité du marché, l'intensité de la demande des consommateurs pour le produit et le choix du réseau de distribution ;

- la coordination et la coopération, qui reflètent la capacité des entreprises à développer une gestion collective efficace du produit.

Les scores obtenus pour chacun des critères sont ensuite multipliés entre eux et divisés par quatre.

Le succès observé a été estimé sur la base de l'évaluation faite plus haut.

Il existe une forte corrélation entre le succès calculé et le succès observé (figure 1). Les facteurs de succès identifiés expliquent véritablement le succès des filières que nous avons étudiées. Néanmoins, le succès observé est plus grand que le succès calculé, à l'exception de trois produits. Ceci peut peut-être s'expliquer par le fait que le prix payé au producteur n'est pas un indicateur parfait de la performance des produits et des filières. Cette différence peut dépendre également des facteurs de succès qui pourraient influencer le profit des entreprises ou les prix de vente (qui n'ont malheureusement pas pu être comparés entre toutes les filières à cause du manque de données comparables).

La question n'est pas ici celle du succès absolu des filières, mais plutôt celle de leur succès relatif comparé à la volonté de l'Union européenne de les soutenir au moyen de la réservation des dénominations, dans le but également de promouvoir les intérêts des consommateurs et des zones rurales défavorisées (voir Règlement 2081/92).

\section{2 / Succès social}

En ce qui concerne le succès social, les procédures d'enregistrement cherchent à maintenir ou à promouvoir le développement local, 
Figure 1. Évaluation du succès économique de 21 filières AOC et IGP.

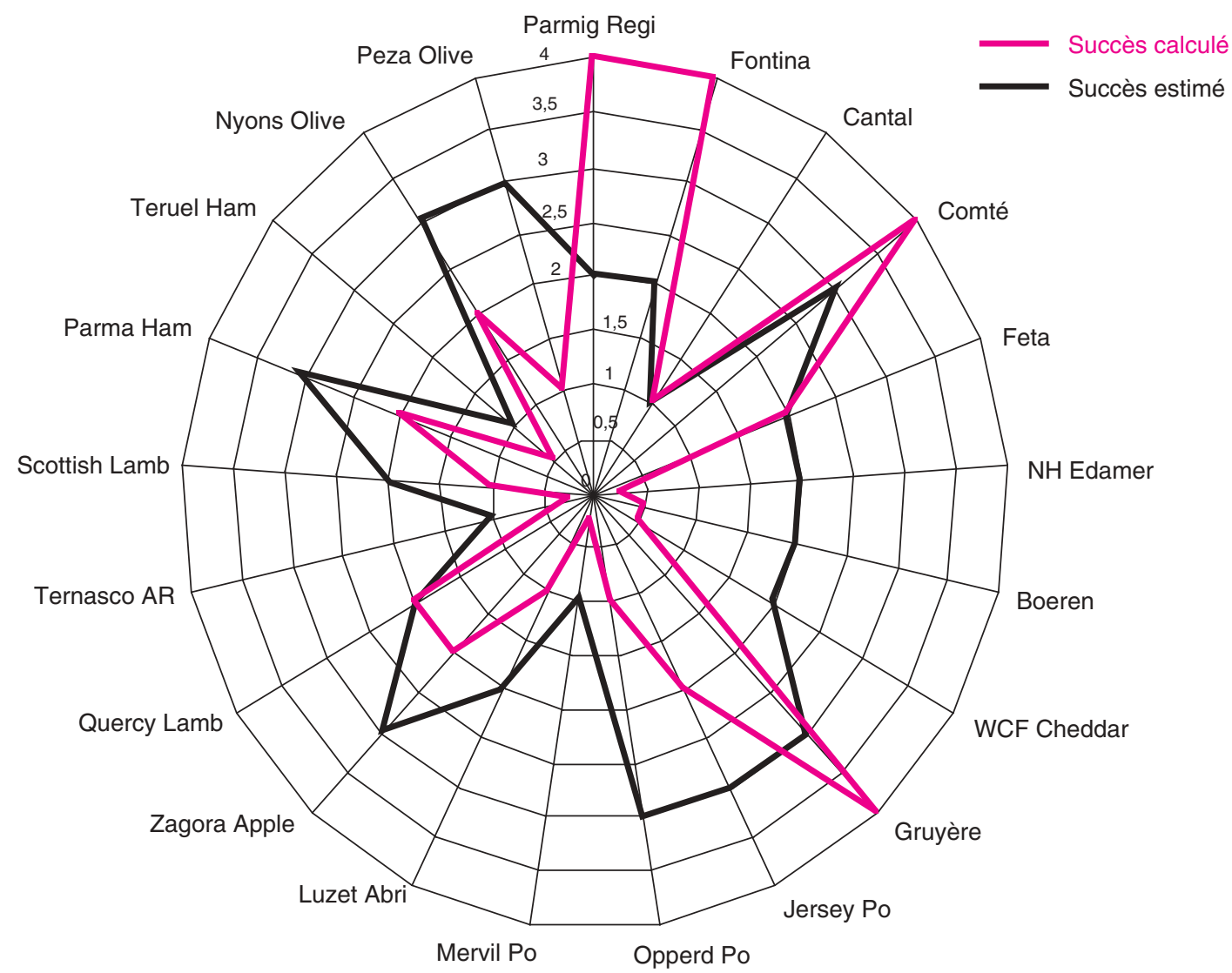

Figure 2. Évaluation du succès social de 21 filières AOC et IGP.

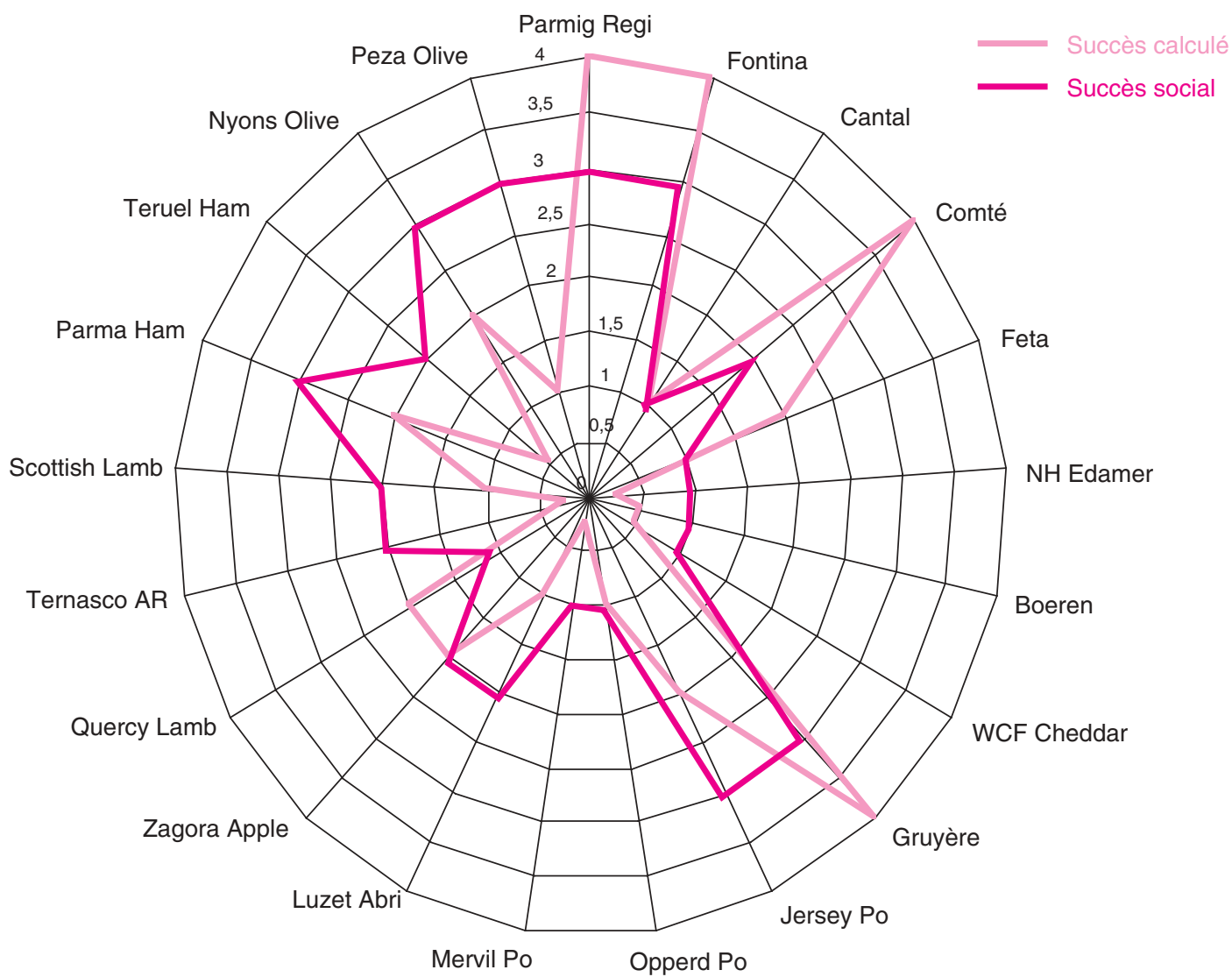


en particulier dans les pays qui ont été les premiers à introduire de telles dispositions (France, Italie et Espagne). De ce point de vue, les filières étudiées ont un impact différent sur les économies régionales. Nous avons attribué un score à chaque produit de 1 à 3 pour un impact faible, modéré ou fort sur l'économie régionale.

A l'exception des quatre produits qui obtiennent les scores les plus élevés (Parmigiano Reggiano, Fontina, Comté, Gruyère), l'impact social est plus important que l'impact économique au sens strict (figure 2). Le rôle social d'une filière est important, malgré des performances économiques modestes, et ne se limite pas aux critères de succès que nous avons utilisés pour définir le succès ni aux prix payés à la production. Ceci justifie pleinement la politique européenne sur les désignations géographiques. Ce phénomène est perceptible dans tous les pays, même dans ceux qui commencent seulement à appliquer la législation. Le phénomène pourrait s'étendre de manière équilibrée dans toute l'Europe.

\section{Conclusion}

Le premier résultat important est que les catégories de produits facilement identifiables à première vue ne sont pas des caractères discriminants. La nature du produit n'est pas déterminante, même si son importance est grande. Des matières premières peuvent donner lieu à une rémunération élevée malgré des contraintes saisonnières et logistiques, alors que plusieurs produits transformés obtiennent des prix faibles. De la même manière, le pays d'origine n'a qu'une importance relative, même si la tradition de chaque pays en matière de protection des noms géographiques peut jouer un certain rôle. Le nombre d'entreprises dans la filière pourrait être considéré comme un frein à la coordination, mais ce n'est pas le cas.

La deuxième conclusion est que nous ne pouvons pas identifier un facteur unique de succès du produit : une conjonction de facteurs est nécessaire. La spécificité du produit est très importante : le règlement européen associe qualité, caractéristiques du produit et origine géographique. Toute la substance du règlement repose sur cette spécificité. Cependant, plusieurs produits spécifiques n'ont pas de succès. La pertinence du marché est certainement un facteur important, parce qu'elle détermine le comportement d'achat des consommateurs. Néanmoins, plusieurs produits positionnés sur des marchés pertinents peinent à se développer s'ils ne sont pas assez spécifiques. C'est généralement le cas lorsqu'ils doivent faire face à la concurrence de produits substituts qui ont plus de succès (Agneau d'Aragon et Jambon du Teruel). La coordination est bien sûr un facteur très important, parce que c'est à la fois une condition et un résultat de l'entente entre les entreprises. L'origine d'une telle coordination reste fréquemment un mystère et des recherches historiques plus poussées seraient nécessaires pour en expliquer les raisons. La coordination n'est pas un modèle exogène aux entreprises, mais elle doit être vue comme un processus en construction. L'existence d'un «channel captain» facilite la coordination : c'est le cas lorsqu'une seule ou quelques entreprises seulement dominent la filière (Pommes de Zagora, Huile d'olive de Peza, Huile d'olive de Nyons). La coordination est intimement liée aux contraintes imposées par les distributeurs, en particulier sur le marché anglo-saxon pour ce qui concerne les produits standards. Les soutiens publics ne peuvent bien sûr pas être considérés comme un facteur important de succès : ils créent une situation de dépendance pour les entreprises et sont le plus souvent limités dans le temps. Le soutien des institutions publiques est particulièrement utile quand il contribue à créer un cadre favorable pour le développement des entreprises (recherche pour la filière du Jambon de Parme, soutien légal pour la filière de l'Huile d'olive de Nyons).

Le troisième résultat important est que le succès est fondé sur la capacité de plusieurs entreprises à construire une filière spécifique, d'une part en fixant des objectifs cohérents au plan collectif (pour ce qui concerne un niveau de gouvernance territoriale et non pas sectorielle) à partir des compétences individuelles (technologie, savoir-faire, gestion stratégique, innovation, etc.) et, d'autre part, en instituant un contrôle à la fois sévère et flexible sur les fonctions de coordination identifiées plus haut. Contrôle sévère pour que les entreprises se conforment aux règles essentielles définies au plan collectif et contrôle flexible pour que chaque entreprise participe au projet tout en jouissant d'une certaine liberté pour développer sa propre stratégie.

Ce texte est repris de l'article de D. Barjolle et B. Sylvander "Quelques facteurs de succès des "produits d'origine" dans les filières agroalimentaires européennes " paru dans la revue Economies et Sociétés, sept-oct 2002, série Systèmes agroalimentaires n²5, p 1441-1462. Edition Les Presses de l'ISMEA (http://www.ismea.org/). 
